\title{
BMJ Open Protocol of a single group prospective observational study on the diagnostic value of 3T susceptibility weighted MRI of nigrosome-1 in patients with parkinsonian symptoms: the N3iPD study (nigrosomal iron imaging in Parkinson's disease)
}

\author{
Stefan T Schwarz, ${ }^{1,2,3}$ Yue Xing, ${ }^{1,4}$ Saadnah Naidu, ${ }^{1}$ Jim Birchall, ${ }^{5}$ Rob Skelly, ${ }^{6}$ \\ Alan Perkins, ${ }^{1,4,7}$ Jonathan Evans, ${ }^{8}$ Gill Sare, ${ }^{8}$ Antonio Martin-Bastida, ${ }^{9}$ Nin Bajaj, \\ Penny Gowland, ${ }^{7}$ Paola Piccini, ${ }^{9}$ Dorothee P Auer ${ }^{1,7,10}$
}

To cite: Schwarz ST, Xing Y, Naidu S, et al. Protocol of a single group prospective observational study on the diagnostic value of $3 T$ susceptibility weighted MRI of nigrosome- 1 in patients with parkinsonian symptoms: the N3/PD study (nigrosomal iron imaging in Parkinson's disease). BMJ Open 2017;7:e016904. doi:10.1136/ bmjopen-2017-016904

- Prepublication history and additional material for this paper are available online. To view please visit the journal (http:// dx.doi.org/10.1136/bmjopen2017-016904).

YX and SN contributed equally.

Received 24 March 2017 Revised 31 May 2017 Accepted 3 July 2017

CrossMark

For numbered affiliations see end of article.

Correspondence to Professor Dorothee P Auer; dorothee.auer@nottingham. ac.uk

\section{ABSTRACT}

Introduction Parkinson's disease (PD) is the most common movement disorder in the elderly and is characterised clinically by bradykinesia, tremor and rigidity. Diagnosing Parkinson's can be difficult especially in the early stages. High-resolution nigrosome MRI offers promising diagnostic accuracy of patients with established clinical symptoms; however, it is unclear whether this may help to establish the diagnosis in the early stages of $\mathrm{PD}$, when there is diagnostic uncertainty. In this scenario, a single photon emission CT scan using a radioactive dopamine transporter ligand can help to establish the diagnosis, or clinical follow-up may eventually clarify the diagnosis. A non-invasive, cost-effective diagnostic test that could replace this would be desirable. We therefore aim to prospectively test whether nigrosome MRI is as useful as DaTSCAN to establish the correct diagnosis in people with minor or unclear symptoms suspicious for PD. Methods and analysis In a prospective study we will recruit 145 patients with unclear symptoms possibly caused by Parkinson's from three movement disorder centres in the UK to take part in the study. We will record the Movement Disorder Society - Unified Parkinson's Disease Rating Scale, and participants will undergo DaTSCAN and high-resolution susceptibility weighted MRI at a field strength of 3T. DaTSCANs will be assessed visually and semiquantitatively; MRI scans will be visually assessed for signal loss in nigrosome- 1 by blinded investigators. We will compare how the diagnosis suggested by MRI compares with the diagnosis based on DaTSCAN and will also validate the diagnosis based on the two tests with a clinical examination performed at least 1 year after the initial presentation as a surrogate gold standard diagnostic test.

Ethics and dissemination The local ethics commission (Health Research Authority East Midlands - Derby Research Ethics Committee) has approved this study (REC ref.: 16/EM/0229). The study is being carried out
Strengths and limitations of this study

This is a prospective, fully powered study with predefined diagnostic test indices that will be tested against an established imaging test and clinical progression.

- The study lacks postmortem gold standard.

- A 1-year follow-up period limits diagnostic accuracy.

under the principles of the Declaration of Helsinki (64th, 2013) and Good Clinical Practice standards. We have included a number of 15 research-funded DaTSCAN in the research protocol. This is to compensate for study site-specific National Health Service funding for this investigation in affected patients. We therefore have also obtained approval from the Administration of Radioactive Substances Administration Committee (ARSAC Ref 253/3629/35864). All findings will be presented at relevant scientific meetings and published in peer-reviewed journals, on the study website, and disseminated in lay and social media where appropriate.

Trial registration number NCT03022357; Pre-results.

\section{INTRODUCTION}

Background and rationale

Idiopathic Parkinson's disease (PD) is characterised by loss of midbrain dopaminergic neurons preferentially affecting the nigrosomes of the pars compacta of the substantia nigra $(\mathrm{SNpc})$. At the time of clinical diagnosis, an estimated $50 \%-70 \%$ of the neurons of the SNpc are lost. ${ }^{1}$ High-resolution susceptibility weighted MRI (SWI-MRI) at $7 \mathrm{~T}^{2}$ but also $3 \mathrm{~T}^{3}$ can be used to demonstrate probable 
iron-related signal loss of the healthy nigrosome-1 signal in Parkinson's. The loss of the characteristic appearance of the healthy nigrosome ('swallow tail sign') proved to have a high diagnostic accuracy between patients with established PD and controls. ${ }^{2-14}$

What is unclear is whether nigrosome MRI has clinical utility in clinically uncertain cases either in the presence of atypical features or in the very early stages of Parkinson's when only very limited clinical signs of the disease are present. DaTSCAN has been proven clinically useful in these scenarios based on information on the functional integrity of the striatal dopaminergic system, but is not widely available, carries a radiation risk and is costly. In most countries there is a cost-benefit of MRI scans in comparison to DaTSCANs (eg, in the UK DaTSCAN $\sim £ 1000$ per scan).

Nigrosomal MRI would be a desirable alternative as it is non-invasive, widely available, substantially less expensive and provides additional structural information on brain pathologies. This study will prospectively investigate the accuracy of nigrosome MRI in diagnosing Parkinson's in comparison to DaTSCAN and the clinical diagnosis 1 year after the initial presentation.

\section{Purpose}

- to establish whether nigrosome MRI is an alternative to DaTSCAN in the diagnosis of early Parkinson's and clinically uncertain parkinsonian syndromes

- to establish whether nigrosomal iron predicts the severity of Parkinson's.

\section{Primary objective}

Validation of nigrosome MRI as a qualitative diagnostic marker in early Parkinson's and clinically uncertain parkinsonian syndromes.

- to investigate whether the presence or absence of the 'swallow tail sign' on nigrosome MRI at $3 \mathrm{~T}$ is as accurate as DatSCAN and at least $80 \%$ sensitive and $80 \%$ specific to predict the final clinical diagnosis of Parkinson's versus other movement disorder in patients with indeterminate or atypical parkinsonian features

Hypothesis 1: that the 'swallow tail sign' is an accurate marker of early Parkinson's.

\section{Secondary objectives}

Biomarker discovery based on quantitative nigrosome iron markers in Parkinson's.

- to investigate whether diagnostic performance of nigrosome MRI can be further improved through quantitative assessment of nigrosomal iron in combination with neuromelanin metrics

Hypothesis 2: that nigrosomal iron metrics further improve the detection of early Parkinson's.

- to assess how well disease severity can be predicted by quantitative measures of iron content in the nigrosome alone and in combination with metrics of neuromelanin content in the substantia nigra $(\mathrm{SN})$
Hypothesis 3: that nigrosomal iron metrics are associated with severity of early Parkinson's.

\section{METHODS: PARTICIPANT AND INTERVENTIONS OUTCOMES Design}

This is a multicentre observational study conducted at the University of Nottingham, Imperial College London and Royal Derby Hospital (all in UK).

\section{Study setting}

We will recruit 145 patients with unclear symptoms possibly caused by Parkinson's from three movement disorder centres in the UK to take part in the study (Nottingham University Hospitals, Derby Teaching Hospitals NHS Trust or Imperial College Healthcare NHS Trust London). This includes patients with a clinical suspicion of PD, but not clearly meeting the UK Brain Bank diagnostic criteria for PD. We will also include patients with parkinsonism and a high degree of diagnostic uncertainty ('clinically uncertain parkinsonian syndromes'). Participants will be investigated by a DaTSCAN and a dedicated MRI at 3T. We will record clinical PD and PD-related rating scales (Unified Parkinson's Disease Rating Scale of the Movement Disorder Society; MDS-UPDRS) at the time of the MRI scan. Presence or absence of nigrosome-1 will be assessed as previously published ${ }^{3}$ and compared with the findings of semiquantitatively analysed DaTSCAN. ${ }^{15} 16$ We will reassess participants clinically after a minimum period of 12 months following the initial assessment and aim to again record clinical PD and PD-related rating scales (MDS-UPDRS). The clinical diagnosis established at the time of the 1-year follow-up will be compared with the outcome of the DaTSCAN and MRI investigation.

\section{Eligibility criteria participants}

A group of 145 participants with suspected PD and clinical uncertainty of diagnosis will be enrolled in the study.

\section{Inclusion criteria}

- ability to give informed consent

- age $>21$ to $<90$ years

- clinical symptoms suspicious for a diagnosis of PD but clinical uncertainty with regard to a definite diagnosis: - clinical symptoms not meeting all of the required UK Brain Bank diagnostic criteria for the diagnosis of PD

- clinical features not typically associated with PD and therefore raising the possibility of a different type disorder/movement disorder

- referred for a DaTSCAN as part of the National Health Service (NHS) clinical diagnostic work-up to investigate a suspicion for a parkinsonian movement disorder-type disease, or referred for a research DaTSCAN as part of this study for the diagnostic work-up to investigate a suspicion for a parkinsonian movement disorder-type disease. 


\section{Exclusion criteria}

- participants with any known contraindication to MRI such as:

- intracranial aneurysm clips

- cardiac pacemakers and defibrillators

- cochlear implants

- MR-incompatible metal implant or tattoo

- patients with a significant head tremor

- claustrophobia

- pregnant women

- participants who are felt to be unfit for the MRI scan according to the judgement of medically qualified personnel, either on the research team or the patient's clinical team (eg, due to back pain, claustrophobia, acute sickness and so on); this includes patients with signs of impaired temperature regulation such as an extremely high fever

- Participants in which a DatSCAN nuclear medical study cannot be performed due to

- severe allergy to iodide compounds

- thyroid gland dysfunction.

\section{Outcomes}

The primary outcome measure of this study is the sensitivity and specificity of the 'swallow tail sign' versus DaTSCAN to predict the clinical diagnosis of PD at 1 year after the initial presentation.

In addition we will report the sensitivity and specificity of the 'swallow tail sign' to predict DaTSCAN results.

The secondary outcomes are (1) the sensitivity and specificity of quantitative metrics of iron and neuromelanin content in the $\mathrm{SN}$ and (2) the association between these metrics with the severity of Parkinson's symptoms.

\section{Participant timeline}

The first date of enrolment was 24 November 2016.

Eligible participants will be enrolled into the study, and the MRI and DatSCAN will be ideally acquired within 3-6 months of each other. At the time when the participants attend for the MRI, they will be assessed clinically for signs of PD and we will record the MDS-UPDRS, along with other PD-related rating scales. The patients will be followed up after a minimum interval of 12 months from the initial visit when we will perform another clinical assessment to establish a clinical diagnosis at follow-up (figure 1).

\section{Sample size}

The study is powered for precision of the determined sensitivity and specificity of the 'swallow tail sign' on nigrosome MRI. In order to be clinically useful, a minimum accuracy of $80 \%$ sensitivity and $80 \%$ specificity defines the lower border of the CI. Based on reported sensitivity and specificity of nigrosome MRI at 3T SWI $(79 \%-100 \%)^{11} 12$ and our own experience (90\%-100\% sensitivity), we assume 0.90 and at least 0.88 for both sensitivity and specificity for NS1 MRI. Sensitivity and specificity of DaTSCAN are in the region of 0.94 and 0.95 , respectively. ${ }^{15}$ The expected proportion of final diagnosis of Parkinson's in our target population is expected to be approximately $50 \%$, and an equal proportion is expected to have a positive DaTSCAN. Available follow-up is expected for $90 \%$ of cases accounting for technical issues not addressed by repeat scans and loss to follow-up. For sensitivity and specificity of 0.90 for nigrosome MRI, and a true positive/true negative split of 50:50 and 140 complete data sets, the SD will be \pm 0.7 resulting in CI (0.83 to 0.97 ) for both sensitivity and specificity. In case either sensitivity or specificity drops to 0.88 , and a 45:55 split of true positive and negative cases, the lower border of the CI for the less prevalent subgroup would still meet the predefined requirement of sensitivity or specificity (CI 0.80 to 0.96 ).

\section{Recruitment}

Participants will be recruited from neurology, care of the elderly clinics or when they are attending for the DaTSCAN investigation as part of their diagnostic workup. The initial approach will be from a member of the patient's usual care team with brief description of what the study involves. If the patient indicates that he/she may be interested in participating in this study, the patient will be asked to sign a consent form to be contacted again by one of the study investigators (online supplementary file 1). A site-specific investigator or research nurse will then be able to contact the potential participant by phone at a later date. Study information may be handed out to the potential participant at the first contact when discussing the participation in the study or posted when being contacted by the research team (online supplementary files 2 and 3 ).

The site investigator in charge of recruitment will contact the potential participants over the phone using the contact details obtained as above after a minimum of 24 hours from the time of first contact. The investigator will explain the information sheet and all pertinent aspects of the study. The patient will then again be asked if they are happy to participate in the study. It will be explained to the potential participant that entry into the trial is entirely voluntary and that their treatment and care will not be affected by their decision. It will also be explained that they can withdraw at any time, but attempts will be made to avoid this occurrence. In the event of their withdrawal, it will be explained that their data collected so far cannot be erased, and we will seek consent to use the data in the final analyses where appropriate. If the patient is happy to participate, an appointment for the first study visit will be arranged. The completion of the consent form will be done at the beginning of the first study visit.

As this study focuses on movement disorders in the adult population, we do not plan to include children or young adults under the age of 21 .

\section{Study regimen}

The participants will be contacted by an investigator or research nurse after they have been recruited to the 


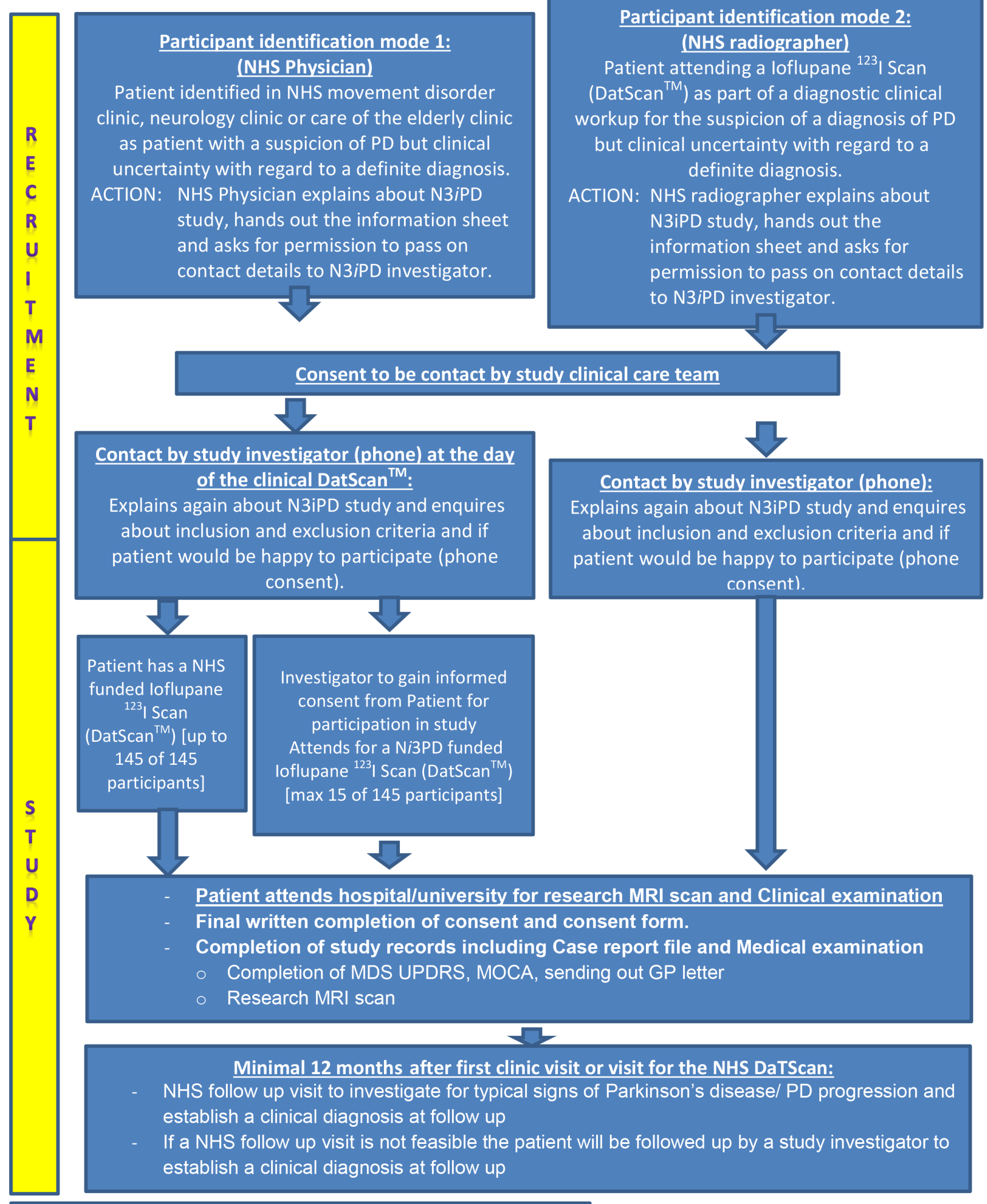

\section{END OF STUDY}

Figure 1 Schematic diagram of time schedule of participant enrolment. GP, general practitioner; MDS-UPDRS, Unified Parkinson's Disease Rating Scale of the Movement Disorder Society; MOCA, Montreal Cognitive Assessment; N3iPD, nigrosomal iron imaging in Parkinson's disease; NHS, National Health Service; PD, Parkinson's disease. 
study to reconfirm that the participant is happy to join the study, and arrangements for study visits will be made. The investigator will discuss the information sheet and all pertinent aspects of the study by phone. If the participant had or will have a DaTSCAN as part of the clinical workup, arrangements will be made for a visit for obtaining written consent, a clinical examination and MRI scan (online supplementary file 4). The clinical examination includes a short physical exam and taking a brief history of allergies, previous diseases and medications. If participants have been started on dopaminergic replacement therapy, they will be instructed to stop these medications in the evening prior to attending a clinical examination/PD-related rating scales (eg, the MDS-UPDRS and Hoehn and Yahr Scale) and to record an MRI scan. The patients will be instructed to take their medication as usual after completion of the study visit. The majority of the included participants are expected not to be on dopamine replacement medication (as very early-stage PD). In case symptomatic treatment has been started, participants are expected to be on a low dose of dopamine replacement therapy as part of the initial treatment or clinical work-up. Omitting the medication in the evening prior to the MRI test is expected to have a small effect, causing a short period of slight deterioration of symptoms of the patients in the morning prior to the study visit. This will be relieved by continuing the normal medication after the study visit on the same day. As stated these patients are usually on small doses of symptom relief dopamine replacement therapy, and omitting the dose prior to the study visit should have only minor impact on patients' symptoms.

If the participant will have a DaTSCAN as part of this study (maximum of 15 of the 145 participants, Queen's Medical Centre, Nottingham University Hospitals NHS Trust only), we will invite the patient to attend for a separate visit. This research DaTSCAN will be performed in place of the 'usual care' and not in addition to the NHS DaTSCAN. All standard NHS procedures will be followed for obtaining the DaTSCAN. Informed consent will be obtained before any research procedures will be undertaken or any research data will be recorded (online supplementary file 5). After attendance to the DaTSCAN, the participant will be invited for a further visit like all the other participants in order to perform a clinical examination/PD-related rating scales and to record an MRI scan. The reasons for the clinical uncertainty of PD and/ or DaTSCAN referral will be documented.

All participants will be followed up after a minimum period of 12 months starting from the NHS clinic visit or DaTSCAN (whichever is earlier). The minimum interval of 12 months between initial study visit and follow-up is necessary to investigate for worsening of clinical symptoms of PD in this time period, which would indicate that the patient has a true diagnosis of PD (final diagnosis at follow-up). The follow-up will be performed either as part of their usual NHS care by their usual NHS doctor or as part of a further study visit if the NHS follow-up is not scheduled for the participant. If feasible the participants will again be asked to stop their dopaminergic replacement therapy in the evening prior to the clinic visit. This will help to more accurately investigate the clinical symptoms as symptom-relieving dopamine replacement therapy makes the clinical examination for signs of PD more unreliable and inaccurate. During the follow-up visit, patients will again be clinically assessed for signs of $\mathrm{PD}$, and if feasible we will record PD-related rating scales (eg, the MDS-UPDRS and Hoehn and Yahr Scale). All clinical data will be recorded and stored as part of the Case Report Form (CRF).

\section{METHODS: DATA COLLECTION, MANAGEMENT AND ANALYSIS Records \\ Case report forms}

Each participant will be assigned a trial identity code number, allocated at randomisation if appropriate, for use on CRFs, other trial documents and the electronic database. The documents and database will also use their initials (first and last names separated by a hyphen or a middle name initial when available) and date of birth $(\mathrm{dd} / \mathrm{mm} / \mathrm{yy})$.

CRFs will be treated as confidential documents and held securely in accordance with regulations. The investigator will make a separate confidential record of the participant's name, date of birth, local hospital number or NHS number and Participant Trial Number (the Trial Recruitment Log) to permit identification of all participants enrolled in the trial, in accordance with regulatory requirements and for follow-up as required.

CRFs shall be restricted to those personnel approved by the chief or local principal investigator and recorded on the 'Trial Delegation Log'.

All paper forms shall be filled in using black ballpoint pen. Errors shall be lined out but not obliterated by using correction fluid, and the correction inserted will be initialled and dated.

The chief or local principal investigator shall sign a declaration ensuring accuracy of data recorded in the CRF.

\section{Sample labelling}

Each participant will be assigned a trial identity code number for use on the samples, consent forms and other study documents and the electronic database. The documents and database will also use their initials (first and last names separated by a hyphen or a middle name initial when available) and date of birth (dd/mm/yy).

\section{Source documents}

Source documents shall be filed at the investigator's site and may include, but are not limited to, consent forms, current medical records, laboratory results and records. A CRF may also completely serve as its own source data. Only trial staff as listed on the Delegation Log shall have 
access to trial documentation other than the regulatory requirements listed below.

\section{Direct access to source data/documents}

The CRF and all source documents, including progress notes and copies of laboratory and medical test results, shall be made available at all times for review by the chief investigator, sponsor's designee and inspection by relevant regulatory authorities (eg, Department of Health (DH), Human Tissue Authority).

\section{Data protection}

All trial staff and investigators will endeavour to protect the rights of the trial's participants to privacy and informed consent, and will adhere to the Data Protection Act, 1998. The CRF will only collect the minimum required information for the purposes of the trial. CRFs will be held securely, in a locked room, or locked cupboard or cabinet. Access to the information will be limited to the trial staff and investigators and relevant regulatory authorities (see above). Computer-held data including the trial database will be held securely and password-protected. All data will be stored on a secure dedicated web server. Access will be restricted by user identifiers and passwords (encrypted using a one-way encryption method).

Information about the trial in the participant's medical records/hospital notes will be treated confidentially in the same way as all other confidential medical information. Electronic data will be backed up every 24 hours to both local and remote media in encrypted format.

\section{Record retention and archiving}

In compliance with The International Council for Harmonisation of Technical Requirements for Pharmaceuticals for Human use (ICH)/Good Clinical Practice (GCP) guidelines, regulations and in accordance with the University of Nottingham Research Code of Conduct and Research Ethics, the chief or local principal investigator will maintain all records and documents regarding the conduct of the study. These will be retained for at least 7 years or for longer if required. If the responsible investigator is no longer able to maintain the study records, a second person will be nominated to take over this responsibility. The Trial Master File and trial documents held by the chief investigator on behalf of the sponsor shall be finally archived at secure archive facilities at the University of Nottingham. This archive shall include all trial databases and associated meta-data encryption codes.

After the completion of this study, we will also pass on anonymised research data (clinical and imaging) to the Michael J Fox Foundation (MJFF), which has funded this study and is based in USA. To further ensure that the participants will also not be identifiable by chance from more refined reconstructions of parts of the head MRI scans, the skull and potentially identifiable facial features from the images will be removed. The research data will then be stored/analysed on databases held by the MJFF or by researchers and research institutes affiliated to the
MJFF. Local data handling, security and access procedures will be implemented by the MJFF.

Anonymised research data generated by this study may be stored by MJFF for up to 7 years and longer if the the potential benefit of making the research data available outweighs the cost of maintaining the database by MJFF.

\section{Statistical methods}

The SWI and Neuromelanin (NM) images will first be visually inspected using three-dimensional reformatting function on the OsiriX platform at the University of Nottingham. More advanced data analysis such as generating the quantitative susceptibility map based on SWI and semiautomated NM quantitation will be performed using scripts coded in Matlab and Unix system. Up-todate, standard structural and functional MRI analysis software tools like Statistical Parametric Mapping (SPM) and FSL and FreeSurfer will be applied to facilitate the relevant data processing. Analysis of other structural and metabolic MR scans will be performed on standard NHS Integrated Primary and Acute Care Systems (PACS) workstations. The further, data analysis of data recorded from the different research centres in London may be analysed locally. This analysis will be performed after anonymisation on university or NHS computers by the primary investigator or trained delegates. For statistical analysis, software tools like SPSS and Microsoft Excel will be used. We will perform an interim analysis of the recorded MRI and DaTSCANs in month 12-18 of the study to compare the sensitivity of the two techniques in the recruited subjects within the first part of the study period. The results of the interim analysis will be communicated and discussed with the funding party (MJFF). There are no planned stopping boundaries with regard to the results of the interim analysis.

\section{Assessment of efficacy}

Analysis for presence or absence of the 'swallow tail sign' as diagnostic marker for PD pathology will be performed as previously published ${ }^{7}$ :

Nigrosome-1 is located in the posterior third of the $\mathrm{SN}$, returns a high signal on SWI in a 'linear', 'comma' or 'wedge' shape, and is surrounded by low SWI signal intensity anterior and laterally (pars compacta $\mathrm{SN}$ ) and medially by low signal from the medial lemniscus.

Presence of the swallow tail/nigrosome-1 will be rated for each hemimesencephalon at the level of the caudal posterior $\mathrm{SN}$ on axial and reformatted scans by at least two raters individually. Given the asymmetrical onset of PD, unilateral absence of nigrosome-1 will be classified as indicative of PD irrespective of the presence or absence of nigrosome-1 on the other side. Reproducibility of nigrosome scoring for inter-rater and intrarater ( $>4$ weeks' interval between image analysis) variability will be tested by calculation of absolute and kappa statistics. Consensus agreement for cases that are scored differently by the two investigators will be sought in a final assessment. The reasons for clinical uncertainty of PD and/or 
DaTSCAN referral will be collated and examined in the final assessment.

\section{METHODS: MONITORING}

\section{Data monitoring}

Monitoring of trial data shall include confirmation of informed consent; source data verification; data storage and data transfer procedures; local quality control checks and procedures, back-up and disaster recovery of any local databases; and validation of data manipulation. The trial coordinator/academic supervisor, or where required, a nominated designee of the sponsor, shall carry out monitoring of trial data as an ongoing activity.

Entries on CRFs will be verified by inspection against the source data. A sample of CRFs $(10 \%$ or as per the study risk assessment) will be checked on a regular basis for verification of all entries made. In addition the subsequent capture of the data on the trial database will be checked. Where corrections are required, these will carry a full audit trail and justification.

Trial data and evidence of monitoring and systems audits will be made available for inspection by Research Ethics Committees (REC) as required.

\section{Potential adverse effects in this study}

The performed MRI investigation is not expected to cause significant pain or discomfort to the participant. Therefore no formal advice for pain relief will be given to the participants.

\section{MRI scanner adverse events}

Using standard safety screens for contraindications, for example, metallic implants, no adverse events are expected as a result of the MRI scan.

\section{DaTSCAN adverse events}

Any adverse events as a result of the DaTSCAN will be followed up in accordance with usual NHS procedures. Serious adverse events will be prevented by carefully considering the contraindications and following local NHS protocols.

\section{Reporting of adverse events}

All adverse events will be recorded and closely monitored until resolution and stabilisation, or until it has been shown that the study procedure is not the cause. The chief investigator shall be informed immediately of any serious adverse events and shall determine seriousness and causality in conjunction with any treating medical practitioners.

\section{Auditing}

Trial conduct may be subject to systems audit of the Trial Master File for inclusion of essential documents; permissions to conduct the trial; Trial Delegation Log; Curriculum Vitaes (CVs) of trial staff and training received; local document control procedures; consent procedures and recruitment logs; adherence to procedures defined in the protocol (eg, inclusion/exclusion criteria, correct randomisation, timeliness of visits); adverse event recording and reporting; and accountability of trial materials and equipment calibration logs.

\section{Ethics and dissemination}

The institutional research ethics committee (Health Research Authority East Midlands - Derby Research Ethics Committee) of the University Hospitals of Nottingham has approved the study (REC number: 16/EM/0229). We have also obtained approval from the Health Research Authority (Integrated Research Application System (IRAS) ) project ID: 198586) and the respective research and development boards. We have obtained approval from the Administration of Radioactive Substance Advisory Committee (ARSAC Ref 253/3629/35864). The study is supported by the local clinical research network (Trent-CLRN). Information about the study will be provided to participants by REC-approved patient information sheets.

\section{Ethical and regulatory aspects}

Performance of the MRI scan

As long as the participant has no contraindication to MRI, for which participants will be screened prior to scanning, MRI scans are widely accepted as being safe. If uncomfortable transient symptoms develop during a scan, such as vertigo or dizziness (occasionally associated with sudden movements within the scanner), the scan can be interrupted.

\section{Unexpected findings during examinations}

Site-specific local standard operating procedures will be followed for dealing with unexpected incidental findings and dealing with previously uncovered potentially treatable or non-treatable medical conditions. Potentially uncovering significant incidental findings could have significant future implications to the participant. This possibility of uncovering medical conditions and resulting future implications is explained in the participant information sheet.

Research MRIs will not be routinely reviewed by trained NHS neuroradiologists and therefore abnormalities recorded on the research MRI may not be uncovered. This will be explained as part of the participant information sheet. Despite this, each participant will have to give written consent agreeing that the general practitioner (GP) or responsible clinician will be informed about the participation in this study. Each participant will have to agree that uncovered incidental findings may be communicated to the GP or responsible clinician to arrange for an appropriate course of action or further tests if needed. In the event of uncovering a relevant incidental finding, a radiological report will be transmitted to the local responsible researcher who will inform the participant and their GP.

\section{Informed consent and participant information}

The process for obtaining participant information will be in accordance with the REC guidance and GCP and 
any other regulatory requirements that might be introduced. The investigator or their nominee and the participant shall both sign and date the informed consent form before the person can participate in the study.

The participant will receive a copy of the signed and dated forms, and the original will be retained in the Trial Master File. A second copy will be filed in the participant's medical notes, and a signed and dated note made in the notes that informed consent was obtained for the trial.

The decision regarding participation in the study is entirely voluntary. The investigator or their nominee shall emphasise to them that consent regarding study participation may be withdrawn at any time without penalty or affecting the quality or quantity of their future medical care, or loss of benefits to which the participant is otherwise entitled. No trial-specific interventions will be done before informed consent has been obtained.

The investigator will inform the participant of any relevant information that becomes available during the course of the study, and will discuss with them whether they wish to continue with the study. If applicable they will be asked to sign revised consent forms.

If the informed consent form is amended during the study, the investigator shall follow all applicable regulatory requirements pertaining to approval of the amended informed consent form by the REC and use of the amended form (including for ongoing participants).

\section{Access to data}

Data access to the research data may be granted to the coinvestigators of the different research sites. Anonymised research data will be transferred to the funder (MJFF) after completion of the study and may be then made available to the wider research community.

\section{DISCUSSION}

To our best knowledge this will be the first prospective study to assess the clinical utility of nigrosome MRI using high-resolution SWI-MRI at 3T in patients with unclear symptoms possibly caused by Parkinson's. We hypothesise that SWI-MRI is as accurate as the only clinically approved test called DaTSCAN, which is a nuclear medical study that involves injection of a radioactive tracer, is commonly more expensive than MRI and has limited availability across many countries. We will assess the diagnostic accuracy of SWI and DatSCAN in comparison to the clinical diagnosis at follow-up (after 12 months) as a surrogate gold standard test.

A limitation of this study is that we do not have a confirmation of the disease status by the ideal gold standard diagnostic test of a postmortem investigation. A further limitation is limited participant number, which will allow us to compare the accuracy of the different diagnostic tests; however, it would not allow us to statistically prove non-inferiority of SWI-MRI when compared with DaTSCAN. However, comparing the two tests with the gold standard diagnosis at 1-year follow-up will ensure that our results are valid and relevant. As a longer follow-up period enhances diagnostic accuracy, the shorter 1-year follow-up period is recognised as another limitation. However, this follow-up period is acceptable for the current study as it will be adequate to diagnose the vast majority of patients. In addition, the power calculation allows for this. 17

If we can show that nigrosome MRI is as useful as DatSCAN to diagnose Parkinson's, this will pave the way for its clinical use. The main benefit of an MRI PD test would be that more people affected by unclear movement disorder symptoms that are suspicious for PD will benefit from an earlier accurate diagnosis, which in turn will help to ensure that patients receive the best possible symptomatic relief treatment earlier.

\section{Author affiliations}

${ }^{1}$ Radiological Sciences, Division of Clinical Neuroscience, School of Medicine, University of Nottingham, Nottingham, UK

${ }^{2}$ Department of Radiology, Cardiff and Vale University Health Board, Cardiff University Hospitals, Cardiff, UK

${ }^{3}$ Cardiff University Brain Research Imaging Centre (CUBRIC), University of Cardiff, Cardiff, UK

${ }^{4}$ Nottingham University Hospitals, Department of Nuclear Medicine, Queen's Medical Centre, Nottingham, UK

${ }^{5}$ Department of Nuclear Medicine, Derby Teaching Hospitals NHS Foundation Trust, Derby, UK

${ }^{6}$ Department of Elderly Medicine, Derby Teaching Hospitals NHS Foundation Trust, Derby, UK

${ }^{7}$ Sir Peter Mansfield Imaging Centre, University of Nottingham, Nottingham, UK ${ }^{8}$ Department of Neurology, Nottingham University Hospitals, Queen's Medical Centre, Nottingham, UK

${ }^{9}$ Centre for Neuroinflammation and Neurodegeneration, Imperial College London, London, W12 0NN, UK

${ }^{10}$ Nottingham NIHR Biomedical Research Centre, Nottingham University Hospital Trusts and University of Nottingham, Nottingham, UK

Acknowledgements The authors thank the efforts of Sharon Forman, Linda Pycroft, Alex Berry, David Sauvain, Samantha Lewis (nee: Bateman), Angela Shone, Tiffany Hamilton and the NHS R\&l support teams (Lauren Blackburn and Jo Burns).

Contributors DPA and STS designed the study, and DPA, STS, YX and PP developed the research protocol, which was edited by NB and PG. Representatives of the sponsor reviewed and edited the protocol (Samantha Lewis). SN will coordinate the study, is responsible for governance, patient recruitment at Nottingham, data acquisition, and will conduct image and statistical analyses, supported by YX. JB, AP, PP and AM-B will perform the DatSCAN acquisition and analysis. JB, RS, AP, JE, GS, NB, PP and AM-B will undertake recruitment at Derby and Imperial. STS, DPA and SN wrote the manuscript. All authors approved the final manuscript.

Funding The work is supported by Michael J Fox Foundation (Grant ID: 11473). Study sponsor: University of Nottingham. Sponsor's reference: 13124 . Contact name: Angela Shone. Address: Room B50, King's Meadow Campus, University of Nottingham, Lenton Lane, Nottingham, NG7 2NR, UK.

Competing interests None declared.

Ethics approval Health Research Authority East Midlands (REC reference: 16/ $\mathrm{EM} / 0229)$.

Provenance and peer review Not commissioned; externally peer reviewed.

Open Access This is an Open Access article distributed in accordance with the Creative Commons Attribution Non Commercial (CC BY-NC 4.0) license, which permits others to distribute, remix, adapt, build upon this work non-commercially, and license their derivative works on different terms, provided the original work is 
properly cited and the use is non-commercial. See: http://creativecommons.org/ licenses/by-nc/4.0/

(C) Article author(s) (or their employer(s) unless otherwise stated in the text of the article) 2017. All rights reserved. No commercial use is permitted unless otherwise expressly granted.

\section{REFERENCES}

1. Fearnley JM, Lees AJ. Ageing and Parkinson's disease: substantia nigra regional selectivity. Brain 1991;114:2283-301.

2. Blazejewska AI, Schwarz ST, Pitiot A, et al. Visualization of nigrosome 1 and its loss in PD: pathoanatomical correlation and in vivo 7 T MRI. Neurology 2013;81:534-40.

3. Schwarz ST, Afzal M, Morgan PS, et al. The 'swallow tail' appearance of the healthy nigrosome - a new accurate test of Parkinson's disease: a case-control and retrospective cross-sectional MRI study at 3T. PLoS One 2014;9:e93814.

4. Cosottini M, Frosini D, Pesaresi I, et al. MR Imaging of the Substantia Nigra at 7 T Enables Diagnosis of Parkinson Disease. Radiology 2014;271:831-8.

5. Cosottini M, Frosini D, Pesaresi I, et al. Comparison of 3T and 7T susceptibility-weighted angiography of the substantia nigra in diagnosing Parkinson disease. AJNR Am J Neuroradiol 2015;36.

6. Reiter E, Mueller C, Pinter B, et al. Dorsolateral nigral hyperintensity on 3.0T susceptibility-weighted imaging in neurodegenerative Parkinsonism. Mov Disord 2015;30:1068-76.

7. Nam Y, Gho SM, Kim DH, et al. Imaging of nigrosome 1 in substantia nigra at 3T using multiecho susceptibility map-weighted imaging (SMWI). J Magn Reson Imaging 2017;46.

8. Gao P, Zhou PY, Li G, et al. Visualization of nigrosomes-1 in 3T MR susceptibility weighted imaging and its absence in diagnosing Parkinson's disease. Eur Rev Med Pharmacol Sci 2015;19:4603-9.
9. Gao P, Zhou PY, Wang PQ, et al. Universality analysis of the existence of substantia nigra "swallow tail" appearance of nonParkinson patients in 3T SWI. Eur Rev Med Pharmacol Sci 2016;20:65-37.

10. Fu KA, Nathan R, Dinov ID, et al. T2-imaging changes in the nigrosome- 1 relate to clinical measures of parkinson's disease. Front Neurol 2016;7:174.

11. Cosottini M, Frosini D, Pesaresi I, et al. Comparison of $3 T$ and 7T susceptibility-weighted angiography of the substantia nigra in diagnosing Parkinson disease. AJNR Am J Neuroradiol 2015;36:461-6.

12. Sung $\mathrm{YH}$, Noh $\mathrm{Y}$, Lee J, et al. Drug-induced Parkinsonism versus Idiopathic Parkinson Disease: Utility of Nigrosome 1 with 3-T Imaging. Radiology 2016;279:151466.

13. Noh Y, Sung YH, Lee J, et al. Nigrosome 1 detection at $3 T$ MR for the diagnosis of early-stage idiopathic parkinson disease: assessment of diagnostic accuracy and agreement on imaging asymmetry and clinical laterality. AJNR Am J Neuroradiol 2015;36:2010-6.

14. Lehéricy S, Bardinet E, Poupon C, et al. 7 Tesla magnetic resonance imaging: a closer look at substantia nigra anatomy in Parkinson's disease. Mov Disord 2014;29:1574-81.

15. Hauser R A, Bajaj N, Kenneth M, et al. Sensitivity, Specificity, Positive and Negative Predictive Values and Diagnostic Accuracy of DaTscan (lofluopane I123 Injection): Predicting Clinical Diagnosis in Early Clinically Uncertain Parkinsonian Syndrome. Journal of Neurology \& Stroke 2014;1:1-13.

16. Bajaj N, Hauser RA, Grachev ID. Clinical utility of dopamine transporter single photon emission CT (DaT-SPECT) with (123I) ioflupane in diagnosis of parkinsonian syndromes. J Neurol Neurosurg Psychiatry 2013;84:1288-95.

17. Tolosa E, Borght TV, Moreno E, et al. Accuracy of DaTSCAN (123I-loflupane) SPECT in diagnosis of patients with clinically uncertain parkinsonism: 2-year follow-up of an open-label study. Mov Disord 2007;22:2346-51. 